\title{
TITLE:
}

\section{Glyoxalase system in yeasts: structure, function, and physiology.}

\section{$\operatorname{AUTHOR}(\mathrm{S})$ :}

Inoue, Yoshiharu; Maeta, Kazuhiro; Nomura, Wataru

\section{CITATION:}

Inoue, Yoshiharu ...[et al]. Glyoxalase system in yeasts: structure, function, and physiology.. Seminars in cell \& developmental biology 2011, 22(3): 278-284

ISSUE DATE:

2011-05

URL:

http://hdl.handle.net/2433/142511

RIGHT:

(c) 2011 Elsevier Ltd. 
Glyoxalase system in yeasts: structure, function, and physiology

Yoshiharu Inoue*, Kazuhiro Maeta ${ }^{\ddagger}$, Wataru Nomura

Laboratory of Molecular Microbiology, Division of Applied Life Sciences, graduate School of Agriculture, Kyoto University, Uji, Kyoto 611-0011, Japan

* Corresponding author. Tel.: +81 774-38-3773; fax.: +81 774-38-3789.

E-mail address: y_inoue@kais.kyoto-u.ac.jp (Y. Inoue)

* Present address: Chemical Genetics Laboratory, RIKEN, Wako, Saitama 351-0198, Japan. 


\section{ABSTRACT}

The glyoxalase system consists of glyoxalase I and glyoxalase II. Glyoxalase I catalyzes the conversion of methylglyoxal $\left(\mathrm{CH}_{3} \mathrm{COCHO}\right)$, a metabolite derived from glycolysis, with glutathione to $S$-D-lactoylglutathione, while glyoxalase II hydrolyses this glutathione thiolester to D-lactic acid and glutathione. Since methylglyoxal is toxic due to its high reactivity, the glyoxalase system is crucial to warrant the efficient metabolic flux of this reactive aldehyde. The budding yeast Saccharomyces cerevisiae has the sole gene (GLO1) encoding the structural gene for glyoxalase I. Meanwhile, this yeast has two isoforms of glyoxalase II encoded by GLO2 and GLO4. The expression of GLO1 is regulated by Hog1 mitogen-activated protein kinase and Msn2/Msn4 transcription factors under highly osmotic stress conditions. The physiological significance of $G L O 1$ expression in response to osmotic stress is to combat the increase in the levels of methylglyoxal in cells during the production of glycerol as a compatible osmolyte. Deficiency in GLOI in S. cerevisiae causes pleiotropic phenotypes in terms of stress response, because the steady state level of methylglyoxal increases in glol $\Delta$ cells thereby constitutively activating Yap1 transcription factor. Yap1 is crucial for oxidative stress response, although methylglyoxal per se does not enhance the intracellular oxidation level in yeast, but it directly modifies cysteine residues of Yap1 that are critical for the nucleocytoplasmic localization of this b-ZIP transcription factor. Consequently, glyoxalase I can be defined as a negative regulator of Yap1 through modulating the intracellular methylglyoxal level.

Keywords:

Saccharomyces cerevisiae

MAP kinase

Yap1

Stress response

Metabolic signaling 


\section{Contents}

1. Introduction

2. Genes for glyoxalases

2.1. Degradation pathway of methylglyoxal

2.2. GLO1

2.3. GLO2 and GLO4

3. Biochemical aspects of glyoxalases

3.1. Glyoxalase I

3.2. Glyoxalase II

4. Regulation of expression and activity of glyoxalase I

4.1. Osmotic stress response of glyoxalase I

4.1.1. Transcriptional regulation of GLO1 in S. cerevisiae

4.1.2. Posttranslational regulation of SpGlo1 in S. pombe

4.2. Methylglyoxal-induced expression of GLO1

5. Physiological role of glyoxalase I as a regulator for metabolic signaling

5.1. Genome-wide analysis of gene expression in a GLO1-deficient mutant

5.2. Methylglyoxal activates Yap1 transcription factor

6. Concluding remarks

References 


\section{Introduction}

Glycolysis is a ubiquitous and evolutionarily conserved energy-generating system. There is a long history in discovering the enzymes constituting glycolysis, or EMP (Embden-Meyerfoh-Parnas) pathway [1]. In 1913, at that time glycolysis had not yet been established the one that we know today, Carl Neuberg proposed that methylglyoxal is the major intermediate in glycolysis [2]. This hypothesis (methylglyoxal theory) was believed to be true for more than 20 years, but Embden suggested more convincing pathway for production of triosephosphate. Later, Meyerhof and Kiessling demonstrated that methylglyoxal was derived from spontaneous breakdown of glyceraldehyde 3- phosphate [3], and Neuberg's theory was denied. However, not a few numbers of researchers thereafter have attempted to solve the question about the physiological role of methylglyoxal, because this aldehyde inhibits the growth of cells from microorganisms to higher eukaryotes [4,5]. In addition, methylglyoxal is likely to be involved in the pathological process of some diseases, such as diabetes [6,7], Alzheimer disease [8,9], and autism [10].

Yeast has contributed as the excellent model organism of higher eukaryotes to reveal many pivotal and basic biological events. Intracellular methylglyoxal level closely correlates with the activity of glyoxalase I, a ubiquitous enzyme for methylglyoxal metabolism $[11,12]$. If methylglyoxal is involved in the fundamental biological system, the common mechanism must underlie between yeasts and higher eukaryotes in terms of physiological function. This review overviews yeast glyoxalases from the biochemical, physiological, and genetic point of views.

\section{Genes for glyoxalases}

\subsection{Degradation pathway of methylglyoxal}

Methylglyoxal $\left(\mathrm{CH}_{3} \mathrm{COCHO}\right)$ is a typical 2-oxoaldehyde in living cells. Since methylglyoxal has two carbonyl groups, this 2-oxoaldehyde is quite reactive, and therefore, it is sometimes referred to as reactive aldehyde species. The detoxification pathway of methylglyoxal has been extensively studied in many organisms [4,5]. The enzyme activity that converts methylglyoxal to lactic acid was first discovered by Neuberg [13]. The glyoxalase system, consisting of glyoxalase I and glyoxalase II, is ubiquitous in all organisms so far examined. The glyoxalase system in baker's yeast was first described by Racker [14]. In this route, methylglyoxal is condensed with glutathione and gives $S$-D-lactoylglutathione by the action of glyoxalase I, after which the glutathione thiolester is hydrolyzed to D-lactic acid and glutathione by glyoxalase II. The alternative route to detoxify methylglyoxal in S. cerevisiae is a 
reduction/oxidation system involving methylglyoxal reductase and lactaldehyde dehydrogenase [4]. Methylglyoxal is reduced to the L-lactaldehyde by NADPH-dependent methylglyoxal reductase [15], after which the L-lactaldehyde is oxidized to L-lactic acid by $\mathrm{NAD}^{+}$-dependent lactaldehyde dehydrogenase [16], of which the activity is linked with $\mathrm{NAD}(\mathrm{P}) \mathrm{H}$ dehydrogenase [17]. In the yeast Hansenula mrakii, methylglyoxal is reduced to acetol by aldehyde reductase and the enzyme partially purified, although this enzyme activity has not been found in $S$. cerevisiae [18].

\subsection{GLOI}

The structural gene for glyoxalase I (GLO1) of S. cerevisiae contained an open reading frame with 326 amino acids. Genetic analysis revealed that GLO1 is not the essential gene, although a mutant defective in GLO1 exhibits hypersensitivity to methylglyoxal. The overexpression of $G L O 1$ in a mutant defective in $\gamma$-glutamylcysteine synthetase $(g \operatorname{sh} 1)$, a rate-limiting enzyme for glutathione synthesis, is not able to restore the growth arrest caused by exogenously added methylglyoxal; whereas in glutathione synthetase-deficient ( $g s h 2)$ mutant, hypersensitivity to methylglyoxal was partially restored by overexpression of GLO1 [19]. In the gsh2-deficient mutant, $\gamma$-glutamylcysteine (an intermediate of glutathione biosynthesis) is accumulated $[20,21]$, which serves as a substrate for Glo1 instead of glutathione. The structural gene for glyoxalase I $\left(\mathrm{glol}^{+}\right)$from the fission yeast Schizosaccharomyces pombe is also identified [22]. The Glo1 of S. pombe (SpGlo1) shares 50\% identity with S. cerevisiae Glo1 in amino acid sequence. The $g l o 1^{+}$gene is dispensable for $S$. pombe for normal growth, although disruption of $\mathrm{glol}^{+}$enhanced susceptibility to methylglyoxal. The hypersensitivity to methylglyoxal of a GLO1 null mutant $($ glol $\Delta)$ of $S$. cerevisiae was suppressed by glo1 ${ }^{+}$[22] .

The genome sequence project of A. oryzae revealed that this filamentous fungi contains one glyoxalase I homologous gene on chromosome 2. The putative glyoxalase I gene contains three introns, and codes for a protein with 318 amino acids, although genetic analysis whether this open reading frame actually encodes glyoxalase I has not yet been done.

The alignment of Glo1 of S. cerevisiae revealed that it consisted of five conserved segments, which are referred to as the regions I, II, III, IV, and V in the N-terminal half, and similar sequences are found in the C-terminal half (regions I', II', III', IV', and V', respectively). Crustal alignment of the amino acid sequence for zinc-binding occurs in region IV, although the identity in amino acid sequence between region IV and region IV' is low (36\%) compared with those between other respective regions (53-68\%) [23]. Since a core motif of region II shows the 
limited homology with plasma glutathione peroxidase from rat and human [24], the region II may be responsible for the binding of glutathione. This structural property is conserved in SpGlo1 also [22].

\subsection{GLO2 and GLO4}

S. cerevisiae has two isoforms of the structural genes for glyoxalase II, GLO2 and GLO4. GLO2 encodes a protein with 274 amino acids, and the molecular weight of the gene product (Glo2) is 31,306, while GLO4 contains an open reading frame with 285 amino acids and the molecular weight of Glo4 is 32,325 [25]. Expression of GLO2 occurs both in glucose and glycerol media, although GLO4 is expressed only in glycerol medium. Both GLO2 and GLO4 are dispensable for normal growth in glucose and glycerol media, although a glo2 $\Delta$ mutant exhibited the increased susceptibility to methylglyoxal, and a glo2 $\Delta g l o 4 \Delta$ mutant was hypersensitive to methylglyoxal as the glol $\Delta$ mutant. S. pombe $g l o 2^{+}$gene has not yet been experimentally identified, although an open reading frame (SPAC824.07) on chromosome 1 consisting of 256 amino acids showed highest similarity with S. cerevisiae Glo2 and Glo4, and an open reading frame (SPCC13B11.03c) on chromosome 3 consisting of 256 amino acids showed the second similarity. The filamentous fungi A. oryzae has an open reading frame (AO090120000408), consisting of 256 amino acids with 8 introns on chromosome 5, encodes a homologue of glyoxalase II, although the genetic identification has not yet been done.

\section{Biochemical aspects of glyoxalases}

\subsection{Glyoxalase I}

Glyoxalase Is have been purified from various organisms. Those from microbial sources are monomeric enzymes with molecular weights of 20,000 to 38,000 , whereas those from plant and animal enzymes have the homodimer structure (molecular weight of a subunit, $21,000)$. Based on the amino acid sequence of GLO1, the molecular weight of glyoxalase I (Glo1) of S. cerevisiae is calculated to be 37,207 . In the in vitro analysis, the $k_{\text {cat }} / K_{\mathrm{m}}$ value of Glo1 is $1.89 \times 10^{7} \mathrm{M}^{-1} \mathrm{~s}^{-1}$ when methylglyoxal and glutathione were used as substrate, and 3.47 $\times 10^{4} \mathrm{M}^{-1} \mathrm{~s}^{-1}$ for methylglyoxal and $\gamma$-glutamylcysteine [19]. The glyoxalase I of S. pombe (SpGlo1) is also a monomeric enzyme with a molecular weight of 34,000 , and $k_{\text {cat }} / K_{\mathrm{m}}$ value is $4.3 \times 10^{7} \mathrm{M}^{-1} \mathrm{~s}^{-1}$ [22]. In the in situ analysis using digitonin-permeabilized S. cerevisiae cells, the kinetic parameters of Glo1 were obtained as follows: $K_{\mathrm{m}}, 0.53 \pm 0.07 \sim 0.62 \pm 0.18 \mathrm{mM}$, and $V,(3.18 \pm 0.16) \sim(2.86 \pm 0.01) \times 10^{-2} \mathrm{mMmin}^{-1}[26]$. 
Glyoxalase I is a metalloenzyme. Mammalian glyoxalase Is are dimer, and each subunit contains one zinc atom [27]. S. cerevisiae Glo1 has been reported to contain one zinc atom per enzyme molecule [28], but Frickel et al. [29] reported that Glo1 contains at least one iron (Fe(II)) per enzyme molecule and one zinc, but the latter can be replaced by manganese. In the case of mammalian glyoxalase I, the EDTA-treated enzyme can be reactivated by mixing with metals in vitro [30]. This was also the case for the SpGlo1, i. e. approximately $70-80 \%$ activity was restored by $0.5 \mathrm{mM}$ zinc $(84.7 \%), 1 \mathrm{mM}$ cobalt $(78.6 \%)$ or $1 \mathrm{mM}$ nickel $(67.9 \%)$. Manganese at $1 \mathrm{mM}$ reactivated EDTA-treated enzyme by 1.8-fold [22].

Relatively higher thermal stability is one of the characteristic properties of SpGlo1. Almost $100 \%$ activity was retained when the purified enzyme was incubated at $60^{\circ} \mathrm{C}$ for up to 4 $\mathrm{h}$ and $50 \%$ inactivation was achieved by incubating the enzyme at $64^{\circ} \mathrm{C}$ for $150 \mathrm{~min}$ or at $65^{\circ} \mathrm{C}$ for $60 \min [22]$.

\subsection{Glyoxalase II}

S. cerevisiae has two isomers of glyoxalase II, Glo2 and Glo4. Subcellular localization of these isomers is different, i.e. Glo2 is present in the cytoplasm, while Glo4 is present in the mitochondrial matrix [25]. Although Glo4 is found in the mitochondrial matrix, Glo1 is substantially present in the cytoplasm, and glyoxalase I activity was not detected from mitochondria [25]. Since $S$-D-lactoylglutathione, a product of glyoxalase I reaction, is the substrate for glyoxalase II, the intrinsic substrate for Glo4 in the mitochondrial matrix has not yet been identified. Heterologous expression of GLO2 in E. coli cells gave the active glyoxalase II enzyme in the soluble fractions. Meanwhile, soluble Glo4 protein was hardly obtained when GLO4 was expressed in E. coli, and consequently, no glyoxalase II activity was detected. However, when a mutant GLO4 that lacks the N-terminal 10 amino acids was expressed in $E$. coli, strong glyoxalase II activity was observed in the soluble fractions [31]. The N-terminal amino acid of the mature Glo4 protein purified from S. cerevisiae cells was Met-11 [31], indicating that the $\mathrm{N}$-terminal 10 amino acids are processed during transportation of Glo4 into the mitochondrial matrix.

The kinetic parameters of the recombinant Glo2 and Glo4 using $S$-D-lactoylglutathione as a substrate were determined: Glo2, $K_{\mathrm{m}}=112 \mu \mathrm{M}, k_{\mathrm{cat}}=979 \mathrm{~s}^{-1}, k_{\mathrm{cat}} / K_{\mathrm{m}}=8.7 \times 10^{6} \mathrm{M}^{-1} \mathrm{~s}^{-1}$; Glo4, $K_{\mathrm{m}}=72.2 \mu \mathrm{M}, k_{\text {cat }}=723 \mathrm{~s}^{-1}, k_{\text {cat }} / K_{\mathrm{m}}=10.0 \times 10^{6} \mathrm{M}^{-1} \mathrm{~s}^{-1}$. These enzymes are active toward $S$-D-mandeloylglutathione also with the catalytic parameters as follows: Glo2, $K_{\mathrm{m}}=16.6$ 
$\mu \mathrm{M}, k_{\mathrm{cat}}=35.1 \mathrm{~s}^{-1}, k_{\mathrm{cat}} / K_{\mathrm{m}}=2.2 \times 10^{6} \mathrm{M}^{-1} \mathrm{~s}^{-1} ; \mathrm{Glo} 4, K_{\mathrm{m}}=36.5 \mu \mathrm{M}, k_{\mathrm{cat}}=25.8 \mathrm{~s}^{-1}, k_{\mathrm{cat}} / K_{\mathrm{m}}=$ $0.71 \times 10^{6} \mathrm{M}^{-1} \mathrm{~s}^{-1}[31]$.

\section{Regulation of expression and activity of glyoxalase I}

\subsection{Osmotic stress response of glyoxalase I}

\subsubsection{Transcriptional regulation of GLO1 in S. cerevisiae}

Genes encoding the stress-inducible proteins contain characteristic cis-acting element in their promoter region, and transcription factor(s) that respond to respective stress stimuli bind to such cis-element, thereby activating transcription of stress responsive genes. In addition to cis-element and transcription factors, stress sensor proteins also play crucial roles. The GLO1 gene has two cis-acting elements, termed STRE (stress response element, 5'-AGGGG-3') in its promoter region $[32,33]$. Generally, the expression of STRE genes is induced by a wide variety of stresses, such as osmotic stress and oxidative stress, as well as heat shock, and these stress signals are focusing on the STRE [34]. Even though the STRE-driven gene, the expression of GLO1 was specifically induced by osmotic stress [32]. Msn2 and $\mathrm{Msn} 4, \mathrm{C}_{2} \mathrm{H}_{2}$-type zinc finger transcription factor, bind to the STRE to enhance the expression of STRE $[35,36]$. The rate of the osmotic stress-induced expression of GLO1 was approximately $20 \%$ and $50 \%$ compared with wild type strain in $m s n 2 \Delta$ and $m s n 4 \Delta$ mutants, respectively. In $m s n 2 \Delta m s n 4 \Delta$ cells, induction of GLO1 expression under highly osmotic conditions was not observed [32]. Hog1 is an orthologue of mammalian p38 mitogen-activated protein kinase (MAPK) in S. cerevisiae. Hog1 is phosphorylated by Pbs2 (MAPK kinase, MAPKK), and Pbs2 is phosphorylated by redundant MAPKK kinases (MAPKKKs), Ssk2 and Ssk22 [37]. The osmotic stress-induced expression of $G L O 1$ was almost completely repressed in a hog $1 \Delta$ or $p b s 2 \Delta$ mutant.

When S. cerevisiae cells encounter the increased osmolarity of environment, the cells induce rapid increase in expression of various kinds of genes. As one of the adaptive responses, yeast cell produces glycerol as a compatible osmolyte [38]. Glycerol-3-phosphate dehydrogenase, encoded by GPD1, is a key enzyme in biosynthesis of glycerol in yeast. Expression of GPD1 under highly osmotic conditions is regulated by Hog1 [38]. Glycerol-3-phosphate dehydrogenase (Gpd1) reduces dihydroxyacetone phosphate to glycerol 3-phosphate in the presence of NADH, after which Gpp2 hydrolyzes glycerol 3-phosphate to glycerol [39]. Dihydroxyacetone phosphate is also a substrate for triosephosphate isomerase, a glycolytic enzyme, and methylglyoxal is synthesized during this enzyme reaction [40-43]. Expression of both of the $H X T 1$ gene (hexose transporter) and the GLK1 gene (glucokinase) 
was enhanced under highly osmotic conditions [44]. Indeed, glucose consumption was increased approximately $30 \%$ by osmotic stress, and subsequently, intracellular concentration of methylglyoxal increased when the cells were exposed to highly osmotic environments [32]. Together, the physiological significance for the osmotic stress-induced expression of GLO1 in $S$. cerevisiae cells is likely to scavenge methylglyoxal efficiently [32].

\subsubsection{Posttranslational regulation of SpGlo1 in S. pombe}

Likewise in S. cerevisiae, glycerol is used as a compatible osmolyte to adapt highly osmotic conditions in the fission yeast $S$. pombe. Consequently, intracellular methylglyoxal levels increase approximately 2-fold following treatment with $0.9 \mathrm{M}$ sorbitol (highly osmotic conditions) [45]. Concomitantly, glyoxalase I (SpGlo1) activity in S. pombe cells under highly osmotic conditions is increased; however, regulatory mechanism of the enhancement of the SpGlo1 activity is independent of the upregulation of $\mathrm{glol}^{+}$expression. The $\mathrm{glol}^{+} \mathrm{mRNA}$ levels in cells exposed to osmotic stress did not increase, even though the SpGlo1 activity was increased. The protein levels of SpGlo1 were substantially unchanged between sorbitol-treated cells and untreated cells, therefore, translational efficiency of $\mathrm{glol}^{+}$mRNA is not likely to be affected under highly osmotic conditions. The Spc1/Sty1 stress-activated protein kinase (SAPK), a family of p38 MAPK, functions as the module to transfer the extracellular stress stimuli in $S$. pombe. Wis1 is a MAPKK that phosphorylates Spc1/Sty1, and Wis4 and Win1 are MAPKKKs that phosphorylate Wis1. The SpGlo1 activity in a mutant defective in $w i s 1^{+}$or $s p c 1^{+}$was still elevated by osmotic stress, although the protein levels of SpGlo1 did not increase. Although the detailed mechanism of this unique phenomenon has remained to be solved, involvement of some molecular chaperone-like proteins that influence the activity of SpGlo1 under highly osmotic conditions has been predicted. A coimmunoprecipitation analysis revealed that proteins with molecular weights of $28,000,25,000$, and 21,000 were found to physically interact with SpGlo1 in S. pombe cells under highly osmotic conditions, however, identification of them has not yet been done [45].

\subsection{Methylglyoxal-induced expression of GLO1}

Expression of GLO1 in S. cerevisiae is induced by osmotic stress to metabolize methylglyoxal that is inevitably produced during glycerol production, although a glol $\Delta$ mutant does not exhibit any growth defect under highly osmotic conditions. Meanwhile, a mutant defective in Hog1 is sensitive to methylglyoxal [46]. Hog1 is activated through phosphorylation 
by Pbs2 MAPKK, and consequently, a $p b s 2 \Delta$ mutant also exhibited susceptibility to methylglyoxal. Hog 1 is accumulated into the nucleus by osmotic stress [36,47] and functions as a component of the transcription machinery $[48,49]$. Upon methylglyoxal stress, Hog 1 is rapidly concentrated in the nucleus, and subsequently, expression of the genes under the control of Hog1, such as GLO1, was induced [46]. Phosphorylation is necessary for the translocation of Hog 1 into the nucleus by osmotic stress [50]. As expected, Hog1 is phosphorylated upon methylglyoxal treatment. The timing of the phosphorylation coincided well with the nuclear localization of Hog 1 following treatment with methylglyoxal. However, methylglyoxal does not cause the morphological changes being characteristic to osmotic stress, such as shrinkage of vacuole.

S. cerevisiae has a bacterial-like two-component system (His-to-Asp phosphorelay system) consisted of Sln1 (osmosensor with histidine kinase activity), Ypd1 (phosphorelay protein), and Ssk1 (response regulator) [51-54]. Sho1 is another osmosensor in S. cerevisiae. These osmosensors function upstream of Hog1-MAPK cascade. Disruption of the Sln1 branch enhanced susceptibility to methylglyoxal, whereas the shols mutant did not exhibit hypersensitivity to methylglyoxal. Hog1 phosphorylation upon methylglyoxal stress did not occur in $s s k 1 \Delta$ cells (Sln1 branch mutant), and consequently, the nuclear localization of Hog1 was not observed in this mutant. Additionally, the methylglyoxal-induced expression of GLO1 was not observed in the $s s k 1 \Delta$ mutant. Neither phosphorylation nor nuclear localization of Hog 1 was seen in the pbs $2 \Delta$ mutant, indicating that the Hog1-MAPK cascade is most likely to be activated through the $\operatorname{Sin} 1$ branch in the presence of methylglyoxal [46]. In addition to Hog1, $m s n 2 \Delta$ cells exhibited increased sensitivity to methylglyoxal, whereas the sensitivity of $m s n 4 \Delta$ cells was more moderate. Msn2 is concentrated in the nucleus when cells are exposed to methylglyoxal (Fig. 1).

\section{Physiological role of glyoxalase I as a regulator for metabolic signaling}

\subsection{Genome-wide analysis of gene expression in a GLO1-deficient mutant}

Since major source of methylglyoxal is glycolysis, intracellular methylglyoxal level is expected to be high when glucose in the medium is abundant. However, the methylglyoxal level peaked at diauxic shift [11], at which growth phase glucose is almost depleted and energy-generating system is shifted from anaerobic glycolysis to aerobic respiration $[55,56]$. The level of methylglyoxal in the glol $\Delta$ mutant of $S$. cerevisiae in a log-phase of growth is approximately two times higher than that in wild type strain. To gain a clue as to the 
physiological role of methylglyoxal comprehensive analysis of gene expression between wild type and glo1 $\Delta$ cells was conducted. As a result, the expression of 587 genes among approximately 5,800 genes in the genome was accelerated (> 2-fold) in the glol $\Delta$ mutant [57]. For example, genes involved in energy metabolism, such as glycogen metabolism and gluconeogenesis, and fatty acid metabolism were induced. In addition, genes involved in stress response and $\mathrm{Ca}^{2+}$ homeostasis were also induced. Ample amount of glucose $(>1 \%)$ remains in the medium at the log-phase of yeast culture; nonetheless, intriguingly, expression of genes, whose expression is repressed when glucose is present, was induced in the glol $\Delta$ mutant.

It has been reported that methylglyoxal levels are higher in diabetic patients compared with healthy individuals [6]. In diabetic mice, of which blood and tissues the level of methylglyoxal is high, expressions of genes involved in gluconeogenesis such as phosphoenolpyruvate carboxykinase (PEPCK) are enhanced [58,59]. Expression of PCK1 coding for PEPCK was enhanced in the yeast glol $\Delta$ mutant also. In addition, DNA microarray analysis revealed that the expression of several genes involved in glycogen metabolism and fatty acid metabolism is accelerated in glol $\Delta$ cells. In diabetic patients, the insufficient action or supply of insulin impairs the translocation of GLUT4-containing vesicle to the plasma membrane thereby resulting in lowering glucose uptake, which in turn leads to glucose starvation in the skeletal muscle cell even though the blood glucose level is high. To overcome the limited supply of glucose, $\beta$-oxidation of fatty acids is induced in the skeletal muscle cells, and gluconeogenesis is induced in liver cells. These metabolic shifts being characteristic in diabetes somehow resemble those in glol $\Delta$ cells of $S$. cerevisiae predicted from the gene expression profile, i.e. glol $\Delta$ cells behave as if cells are starved of glucose even though glucose is sufficiently present around the cell.

\subsection{Methylglyoxal activates Yap1 transcription factor}

Another striking feature of the gene expression profile of glol $\Delta$ cells is that expression of genes involved in stress response is enhanced [57]. The phenotype of the glol $\Delta$ mutant in terms of stress resistance, such as pleiotropic drug resistance (fluconazole, cycloheximide, cadmium, $\mathrm{H}_{2} \mathrm{O}_{2}$ ) and heat shock, supports the expression profile. Since GLO1-deficiency causes a broad range of phenotype, aberrant metabolism of methylglyoxal is most likely to affect the function of transcription factor(s) involved in stress response. Indeed, methylglyoxal activated Yap1, a bZIP transcription factor [11]. Yap1 is a functional homologue of mammalian AP-1 in $S$. cerevisiae [60,61], and is crucial for the stress response [62]. Overexpression of YAP1 confers 
the resistance against pleiotropic and structurally unrelated drugs [63]. Therefore, the phenotype of the glol $\Delta$ mutant can be account for by the constitutive activation of Yap1.

Yap1 is imported into the nucleus by Pse1 (importin) [64], but is rapidly exported from the nucleus by Crm1 (exportin) $[65,66]$. Therefore, under normal conditions, Yap1 is predominantly distributed in the cytoplasm. Yap1 has six cysteines, i.e. three in the $\mathrm{N}$ terminus (N-terminal cysteine-rich domain, $\mathrm{n}-\mathrm{CRD}$ ), and three in the $\mathrm{C}$ terminus (c-CRD). A nuclear export signal (NES) sequence of Yap1 partially overlaps the c-CRD [67-69]. Upon oxidative stress, intramolecular disulfide bonds between n-CRD and c-CRD are formed with the aid of Gpx3 [70,71]. Gpx3, one of the glutathione peroxidase homologues in yeast [72], functions as a redox sensor and transducer for Yap1 [70,71]. Intramolecular disulfide bonds of Yap1 block the interaction between Crm1 and Yap1, which allows Yap1 to reside in the nucleus thereby activating the transcription of its target gene [72]. Since the expression of several Yap1 target genes was upregulated in the glol $\Delta$ mutant, it is most likely that Yap1 is constitutively activated in glol $\Delta$ cells. As expected, Yap1 was concentrated in the nucleus in glol $\Delta$ cells. Similarly, exogenously added methylglyoxal also caused the nuclear localization of Yap1, even in the absence of Gpx3. The nuclear Yap1 was immediately redistributed in the cytoplasm when methylglyoxal was eliminated from the medium [11]. These observations pointed out two important issues, i.e. the methylglyoxal-dependent Yap1 activation is reversible, and reactive oxygen species are not involved in this event.

Methylglyoxal makes adducts with lysine and arginine residues of protein. The c-CRD/NES of Yap1 contains several lysine and arginine residues, and therefore it is conceivable that modification of them with methylglyoxal might abolish the NES function and inhibit interaction with Crm1, leading to the nuclear localization of Yap1 in glols cells or methylglyoxal-treated cells. However, since adducts formed with methylglyoxal and lysine/arginine residues of protein are irreversible, modification of such arginine and/or lysine residues with methylglyoxal is less feasible. On the other hand, site-directed mutagenesis of three cysteine residues within the c-CRD/NES to alanine or threonine abolished the methylglyoxal-dependent Yap1 activation, indicating that these cysteine residues in c-CRD are the direct targets of methylglyoxal. Importantly, cellular oxidation level does not increase in cells treated with methylglyoxal, and the steady state level of cellular oxidation of glol $\Delta$ cells is essentially the same as wild type cells [73]. Furthermore, a Yap1 mutant containing only one cysteine in the c-CRD but deleting b-ZIP region, which cannot form a dimer and therefore intermolecular disulfide bond may be not feasible, is concentrated in the nucleus following 
treatment with methylglyoxal. This construct is constitutively concentrated in the nucleus of glo1 $\Delta$ cells. Taken together, methylglyoxal reversibly modifies cysteine residues of Yap1 in vivo to accelerate its activity, which leads to alter the cellular function [11] (Fig. 2). Basically, the similar regulatory mechanism is conserved in Pap1, a Yap1 homologue in the fission yeast $S$. pombe [74].

\section{Concluding remarks}

Methylglyoxal has much greater potential than glucose to cause protein glycation. This reaction occurs in vivo also, which is linked to the formation of advanced glycation end products (AGEs) [75]. Decrease in the level of Glo1 expression increases the rate of irreversible protein glycation [12], leading to the production of AGE. AGE activates the receptor for AGE (RAGE), and the AGE-RAGE system is believed to be involved in the pathogenic mechanism of diabetic complications [76]. Thus far, voluminous studies regarding the physiological role of methylglyoxal have focused on the aspect of methylglyoxal as the potent inducer for AGE production $[77,78]$. The fate of irreversibly glycated proteins is likely to be the target of the proteasome-dependent degradation [79]. However, we should pay more attention to the fact that methylglyoxal is able to play a role to modulate the cellular function through the reversible modification of proteins that are crucial for cellular function, because reversibility would be one of the most important characteristics of biological mechanism.

\section{References}

1. Barnett JA. A history of research on yeasts 5: the fermentation pathway. Yeast 2003;20: 509-543.

2. Neuberg C, Kerb J. Über zuckerfreie Hefegärungen, XII. Über die Vorgänge bei der Hefegärung. Biochemische Zeitschrift 1913;53:406-419.

3. Meyerhof O, Kiessling W. Über die phosphorylierten Zwischenprodukte und die letzten Phasen der alkoholischen Gärung. Biochemische Zeitschrift 1933;267:313-348.

4. Inoue Y, Kimura A. Methylglyoxal and regulation of its metabolism in microorganisms. Adv Microb Physiol 1995;37:177-227.

5. Kalapos MP. Methylglyoxal in living organisms: Chemistry, biochemistry, toxicology and biological implications. Toxicol Lett 1999;110:145-175. 
6. McLellan AC, Thornalley PJ, Benn J, Sonksen PH. The glyoxalase system in clinical diabetes mellitus and correlation with diabetic complications. Clin Sci 1994;87:21-29.

7. Ahmed N, Babaei-Jadidi R, Howell SK, Beisswenger PJ, Thornalley PJ. Degradation products of proteins damaged by glycation, oxidation and nitration in clinical type 1 diabetes. Diabetologia 2005;48:1590-1603.

8. Chen F, Wollmer MA, Hoerndli F, Münch G, Kuhla B, Rogaev EI, Tsolaki M, Papassotiropoulos A, Götz J. Role for glyoxalase I in Alzheimer's disease. Proc Natl Acad Sci USA 2004;101:7687-7692.

9. Ahmed N, Ahmed U, Thornalley PJ, Hager K, Fleischer GA, Munch G. Protein glycation, oxidation and nitration marker residues and free adducts of cerebrospinal fluid in Alzheimer's dislink to cognitive impairment. J Neurochem 2004;92:255-263.

10. Junaid MA, Kowal D, Barua M, Pullarkat PS, Brooks SS, Pullarkat RK. Proteomic studies identified a single nucleotide polymorphism in glyoxalase I as autism susceptibility factor. Am J Med Genet 2004;131A:11-17.

11. Maeta K, Izawa S, Okazaki S, Kuge S, Inoue Y. Activity of the Yap1 transcription factor in Saccharomyces cerevisiae is modulated by methylglyoxal, a metabolite derived from glycolysis. Mol Cell Biol 2004;24:8753-8764.

12. Thornalley PJ. Glyoxalase I - structure, function and a critical role in the enzymatic defence against glycation. Biochem Soc Trans 2003;31:1343-1348.

13. Neuberg C. The destruction of lactic aldehyde and methylglyoxal by animal organs. Biochem Z 1913;49: 502-506.

14. Racker E. The mechanism of action of glyoxalase. J Biol Chem 1951;90:685-696.

15. Murata K, Fukuda Y, Simosaka M, Watanabe K, Saikusa T, Kimura A. Metabolism of 2-oxoaldehyde in yeasts. Purification and characterization of NADPH-dependent methylglyoxal-reducing enzyme from Saccharomyces cerevisiae. Eur J Biochem 1985;151:631-636.

16. Inoue Y, Watanabe K, Shimosaka M, Saikusa T, Fukuda Y, Murata K, Kimura A. Metabolism of 2-oxoaldehydes in yeasts. Purification and characterization of lactaldehyde dehydrogenase from Saccharomyces cerevisiae. Eur J Biochem 1985;153:243-247.

17. Inoue $\mathrm{Y}$, Kimura A. Purification and some properties of $\mathrm{NAD}(\mathrm{P}) \mathrm{H}$ dehydrogenase from Saccharomyces cerevisiae: contribution to glycolytic methylglyoxal pathway. J Ferment Bioeng 1994;77:557-561.

18. Inoue $\mathrm{Y}$, Ikemoto S, Kitamura K, Kimura A. Occurrence of a NADH-dependent 
methylglyoxal reducing system: conversion of methylglyoxal to acetol by aldehyde reductase from Hansenula mrakii. J Ferment Bioeng 1992;74:46-48.

19. Inoue Y, Kimura A. Identification of the structural gene for glyoxalase I from Saccharomyces cerevisiae. J Biol Chem 1996;271:25958-25965.

20. Grant CM, MacIver FH, Dawes IW. Glutathione synthetase is dispensable for growth under both normal and oxidative stress conditions in the yeast Saccharomyces cerevisiae due to an accumulation of the dipeptide $\gamma$-glutamylcysteine. Mol Biol Cell 1997;8:1699-707.

21. Inoue Y, Sugiyama K, Izawa S, Kimura A. Molecular identification of glutathione synthetase (GSH2) gene from Saccharomyces cerevisiae. Biochim Biophys Acta 1998;1395:315-320.

22. Takatsume Y, Izawa S, Inoue Y. Identification of thermostable glyoxalase I in the fission yeast Schizosaccharomyces pombe. Arch Microbiol 2004;181:371-377.

23. Inoue Y, Kimura A. Glycolytic-methylglyoxal pathway. Molecular evolution and stress response of glyoxalase I in Saccharomyces cerevisiae. Proc Japan Acad 1999;75B:127-132.

24. Ranganathan S, Walsh ES, Godwin AK, Tew KD. Cloning and characterization of human colon glyoxalase-I. J Biol Chem 1993;268:5661-5667.

25. Bito A, Haider M, Hadler I, Breitenbach M. Identification and phenotypic analysis of two glyoxalase II encoding genes from Saccharomyces cerevisiae, GLO2 and GLO4, and intracellular localization of the corresponding proteins. $J$ Biol Chem 1997;272:21509-21519.

26. Martins AM, Mendes P, Cordeiro C, Freire AP. In situ kinetic analysis of glyoxalase I and glyoxalase II in Saccharomyces cerevisiae. Eur J Biochem 2001;268:3930-3936.

27. Aronsson AC, Marmstål E, Mannervik B. Glyoxalase I, a zinc metalloenzyme of mammals and yeast. Biochem Biophys Res Commun 1978;81:1235-1240.

28. Marmstål E, Aronsson AC, Mannervik B. Comparison of glyoxalase I purified from yeast (Saccharomyces cerevisiae) with the enzyme from mammalian sources. Biochem $\mathbf{J}$ 1979;183:23-30.

29. Frickel EM, Jemth P, Widersten M, Mannervik B. Yeast glyoxalase I is a monomeric enzyme with two active sites. J Biol Chem 2001;276:1845-1849.

30. Uotila L, Koivusalo M. Purification and properties of glyoxalase I from sheep liver. Eur J Biochem 1975;52:493-503. 
31. Bito A, Haider M, Briza P, Strasser P, Breitenbach M. Heterologous expression, purification, and kinetic comparison of the cytoplasmic and mitochondrial glyoxalase II enzymes, Glo2p and Glo4p, from Saccharomyces cerevisiae. Protein Expr Purif 1999;17:456-464.

32. Inoue Y, Tsujimoto Y, Kimura A. Expression of the glyoxalase I gene of Saccharomyces cerevisiae is regulated by high osmolarity glycerol mitogen-activated protein kinase pathway in osmotic stress response. J Biol Chem 1998;273:2977-2983.

33. Takatsume Y, Ohdate T, Maeta K, Nomura W, Izawa S, Inoue Y. Calcineurin/Crz1 destabilizes Msn2 and Msn4 in the nucleus in response to $\mathrm{Ca}^{2+}$ in Saccharomyces cerevisiae. Biochem J 2010;427:275-287.

34. Marchler G, Schüller C, Adam G, Ruis H. A Saccharomyces cerevisiae UAS element controlled by protein kinase A activates transcription in response to a variety of stress conditions. EMBO J 1993;12:1997-2003.

35. Martinez-Pastor MT, Marchler G, Schüller C, Marchler-Bauer A, Ruis H, Estruch F. The Saccharomyces cerevisiae zinc finger proteins Msn2p and Msn4p are required for transcriptional induction through the stress response element (STRE). EMBO J 1996;15:2227-2235.

36. Görner W, Durchschlag E, Martinez-Pastor MT, Estruch F, Ammerer G, Hamilton B, Ruis $\mathrm{H}$, Schüller C. Nuclear localization of the $\mathrm{C}_{2} \mathrm{H}_{2}$ zinc finger protein Msn2p is regulated by stress and protein kinase A activity. Genes Dev 1998;12:586-597.

37. Brewster JL, de Valoir T, Dwyer ND, Winter E, Gustin MC. An osmosensing signal transduction pathway in yeast. Science 1993;259:1760-1763.

38. Albertyn J, Hohmann S, Thevelein JM, Prior BA. GPD1, which encodes glycerol-3-phosphate dehydrogenase, is essential for growth under osmotic stress in Saccharomyces cerevisiae, and its expression is regulated by the high-osmolarity glycerol response pathway. Mol Cell Biol 1994;14:4135-4144.

39. Norbeck J, Pahlman AK, Akhtar N, Blomberg A, Adler L. Purification and characterization of two isoenzymes of D,L-glycerol-3-phosphatase from Saccharomyces cerevisiae. Identification of the corresponding GPP1 and GPP2 genes and evidence for osmotic regulation of Gpp2p expression by the osmosensing mitogen-activated protein kinase signal transduction pathway. J Biol Chem 1996;271:13875-13881. 
40. Iyengar R, Rose IA. Liberation of the triosephosphate isomerase reaction intermediate and its trapping by isomerase, yeast aldolase, and methylglyoxal synthase. Biochemistry 1981;20:1229-1235.

41. Pompliano DL, Peyman A, Knowles JR. Stabilization of a reaction intermediate as a catalytic device: definition of the functional role of the flexible loop in triosephosphate isomerase. Biochemistry 1990;29:3186-3194.

42. Richard JP. Kinetic parameters for the elimination reaction catalyzed by triosephosphate isomerase and an estimation of the reaction's physiological significance. Biochemistry 1991;30:4581-4585.

43. Richard JP. Mechanism for the formation of methylglyoxal from triosephosphates. Biochem Soc Trans 1993;21:549-553.

44. Norbeck J, Blomberg A. Metabolic and regulatory changes associated with growth of Saccharomyces cerevisiae in $1.4 \mathrm{M} \mathrm{NaCl}$. Evidence for osmotic induction of glycerol dissimilation via the dihydroxyacetone pathway. J Biol Chem 1997;272:5544-5554.

45. Takatsume $\mathrm{Y}$, Izawa $\mathrm{S}$, Inoue $\mathrm{Y}$. Unique regulation of glyoxalase I activity during osmotic stress response in the fission yeast Schizosaccharomyces pombe: neither the mRNA nor the protein level of glyoxalase I increase under conditions that enhance its activity. Arch Microbiol 2005;183:224-227.

46. Maeta K, Izawa S, Inoue Y. Methylglyoxal, a metabolite derived from glycolysis, functions as a signal initiator of the high osmolarity glycerol-mitogen-activated protein kinase cascade and calcineurin/Crz1-mediated pathway in Saccharomyces cerevisiae. J Biol Chem 2005;280:253-260.

47. Ferrigno P, Posas F, Koepp D, Saito H, Silver PA. Regulated nucleo/cytoplasmic exchange of HOG1 MAPK requires the importin $\beta$ homologs NMD5 and XPO1. EMBO J 1998; 17:5606-5614.

48. Hohmann S. Osmotic stress signaling and osmoadaptation in yeasts. Microbiol Mol Biol Rev 2002;66:300-372.

49. Westfall PJ, Ballon DR, Thorner J. When the stress of your environment makes you go HOG wild. Science 2004;306:1511-1512.

50. Reiser V, Ruis H, Ammerer G. Kinase activity-dependent nuclear export opposes stress-induced nuclear accumulation and retention of Hog1 mitogen-activated protein kinase in the budding yeast Saccharomyces cerevisiae. Mol Biol Cell 1999;10:1147-1161.

51. Ota IM, Varshavsky A. A yeast protein similar to bacterial two-component regulators. 
Science 1993;262:566-569.

52. Maeda T, Wurgler-Murphy SM, Saito H. A two-component system that regulates an osmosensing MAP kinase cascade in yeast. Nature 1994;369:242-245.

53. Maeda T, Takekawa M, Saito H. Activation of yeast PBS2 MAPKK by MAPKKKs or by binding of an SH3-containing osmosensor. Science 1995;269:554-558.

54. Posas F, Wurgler-Murphy SM, Maeda T, Witten EA, Thai TC, Saito H. Yeast HOG1 MAP kinase cascade is regulated by a multistep phosphorelay mechanism in the SLN1-YPD1-SSK1 "two-component" osmosensor. Cell 1996;86:865-875.

55. Haurie V, Perrot M, Mini T, Jenö P, Sagliocco F, Boucherie H. The transcriptional activator Cat8p provides a major contribution to the reprogramming of carbon metabolism during the diauxic shift in Saccharomyces cerevisiae. J Biol Chem 2001;276:76-85.

56. Young ET, Dombek KM, Tachibana C, Ideker T. Multiple pathways are co-regulated by the protein kinase Snf1 and the transcription factors Adr1 and Cat8. J Biol Chem 2003;278:26146-26158.

57. Maeta K. Cellular response to methylglyoxal in Saccharomyces cerevisiae. Ph.D. Thesis, Kyoto University, Kyoto, Japan, 2005.

58. Herzig S, Long F, Jhala US, Hedrick S, Quinn R, Bauer A, Rudolph D, Schutz G, Yoon C, Puigserver P, Spiegelman B, Montminy M. CREB regulates hepatic gluconeogenesis through the coactivator PGC-1. Nature 2001;413:179-183.

59. Yoon JC, Puigserver P, Chen G, Donovan J, Wu Z, Rhee J, Adelmant G, Stafford J, Kahn CR, Granner DK, Newgard CB, Spiegelman BM. Control of hepatic gluconeogenesis through the transcriptional coactivator PGC-1. Nature 2001;413:131-138.

60. Harshman KD, Moye-Rowley WS, Parker CS. Transcriptional activation by the SV40 AP-1 recognition element in yeast is mediated by a factor similar to AP-1 that is distinct from GCN4. Cell 1988;53:321-330.

61. Moye-Rowley WS, Harshman KD, Parker CS. Yeast YAPI encodes a novel form of the jun family of transcriptional activator proteins. Genes Dev 1989;3:283-292.

62. Toone WM, Jones N. AP-1 transcription factors in yeast. Curr Opin Genet Dev 1999;9:55-61.

63. Hertle K, Haase E, Brendel M. The SNQ3 gene of Saccharomyces cerevisiae confers hyper-resistance to several functionally unrelated chemicals. Curr. Genet 1991;19:429-433.

64. Isoyama T, Murayama A, Nomoto A, Kuge S. Nuclear import of the yeast AP-1-like 
transcription factor Yap1p is mediated by transport receptor Pse1p, and this import step is not affected by oxidative stress. J Biol Chem 2001;276:21863-21869.

65. Kuge S, Toda T, Iizuka N, Nomoto A. Crm1 (XpoI) dependent nuclear export of the budding yeast transcription factor yAP-1 is sensitive to oxidative stress. Genes Cells 1998;3:521-532.

66. Yan C, Lee LH, Davis LI. Crm1p mediates regulated nuclear export of a yeast AP-1-like transcription factor. EMBO J 1998;17:7416-7429.

67. Coleman ST, Epping EA, Steggerda SM, Moye-Rowley WS. Yap1p activates gene transcription in an oxidant-specific fashion. Mol Cell Biol 1999;19:8302-8313.

68. Delaunay A, Isnard $\mathrm{AD}$, Toledano $\mathrm{MB} . \mathrm{H}_{2} \mathrm{O}_{2}$ sensing through oxidation of the Yap1 transcription factor. EMBO J 2000;19:5157-5166.

69. Kuge S, Arita M, Murayama A, Maeta K, Izawa S, Inoue Y, Nomoto A. Regulation of the yeast Yap1p nuclear export signal is mediated by redox signal-induced reversible disulfide bond formation. Mol Cell Biol 2001;21:6139-6150.

70. Delaunay A, Pflieger D, Barrault MB, Vinh J, Toledano MB. A thiol peroxidase is an $\mathrm{H}_{2} \mathrm{O}_{2}$ receptor and redox-transducer in gene activation. Cell 2002;111:471-481.

71. Okazaki S, Tachibana T, Naganuma A, Mano N, Kuge S. Multistep disulfide bond formation in Yap1 is required for sensing and transduction of $\mathrm{H}_{2} \mathrm{O}_{2}$ stress signal. Mol Cell 2007;27:675-688.

72. Kuge S, Jones N, Nomoto A. Regulation of yAP-1 nuclear localization in response to oxidative stress. EMBO J 1997;16:1710-1720.

73. Maeta K, Mori K, Takatsume Y, Izawa S, Inoue Y. Diagnosis of cell death induced by methylglyoxal, a metabolite derived from glycolysis, in Saccharomyces cerevisiae. FEMS Microbiol Lett 2005;243:87-92.

74. Takatsume Y, Izawa S, Inoue Y. Methylglyoxal as a signal initiator for activation of the stress-activated protein kinase cascade in the fission yeast Schizosaccharomyces pombe. J Biol Chem 2006;281:9086-9092.

75. Thornalley PJ. Protein and nucleotide damage by glyoxal and methylglyoxal in physiological systems - role in ageing and disease. Drug Metabol Drug Interact 2008;23:125-150.

76. Vander Jagt DL. Methylglyoxal, diabetes mellitus and diabetic complications. Drug Metabol Drug Interact 2008;23:93-124. 
77. Thornalley PJ, Westwood M, Lo TW, McLellan AC. Formation of methylglyoxal-modified proteins in vitro and in vivo and their involvement in AGE-related processes. Contrib Nephrol 1995;112:24-31.

78. Ramasamy R, Yan SF, Schmidt AM. Methylglyoxal comes of AGE. Cell 2006; $124: 258-260$.

79. Du J, Zeng J, Ou X, Ren X, Cai S. Methylglyoxal downregulates Raf-1 protein through a ubiquitination-mediated mechanism. Int J Biochem Cell Biol 2006;38:1084-1091 . 
Figure legends

Fig. 1. Model for activation of Hog1-MAP kinase cascade.

Osmotic stress is sensed by both osmosensors SIn1 and Sho1, although methylglyoxal is sensed by $\operatorname{Sin} 1$. Upon osmotic stress or methylglyoxal stimulation, Hog1 is phosphorylated and translocated into the nucleus. The nuclear Hog1 recruits transcription factors, such as Msn2 and Msn4, to the promoter region, thereby activating transcription of their target genes. STRE, stress response element.

Fig. 2. Schematic model for activation of Yap1.

Yap1 is predominantly distributed in the cytoplasm under normal conditions. Upon oxidative stress, intramolecular disulfide bonds between cysteine residues in the n-CRD and c-CRD are formed with the aid of Gpx3, and consequently Yap1 is concentrated in the nucleus. In the methylglyoxal-dependent Yap1 activation, Gpx3 is dispensable, and methylglyoxal directly and reversibly modifies cysteine residues in the c-CRD, which partially overlaps the NES (nuclear export signal), which leads to the nuclear localization of Yap1. Since Glo1 is crucial for degradation of methylglyoxal, the Glo1-deficiency constitutively activates Yap1, thereby affecting the cellular functions. MG, methylglyoxal. 
Fig. 1

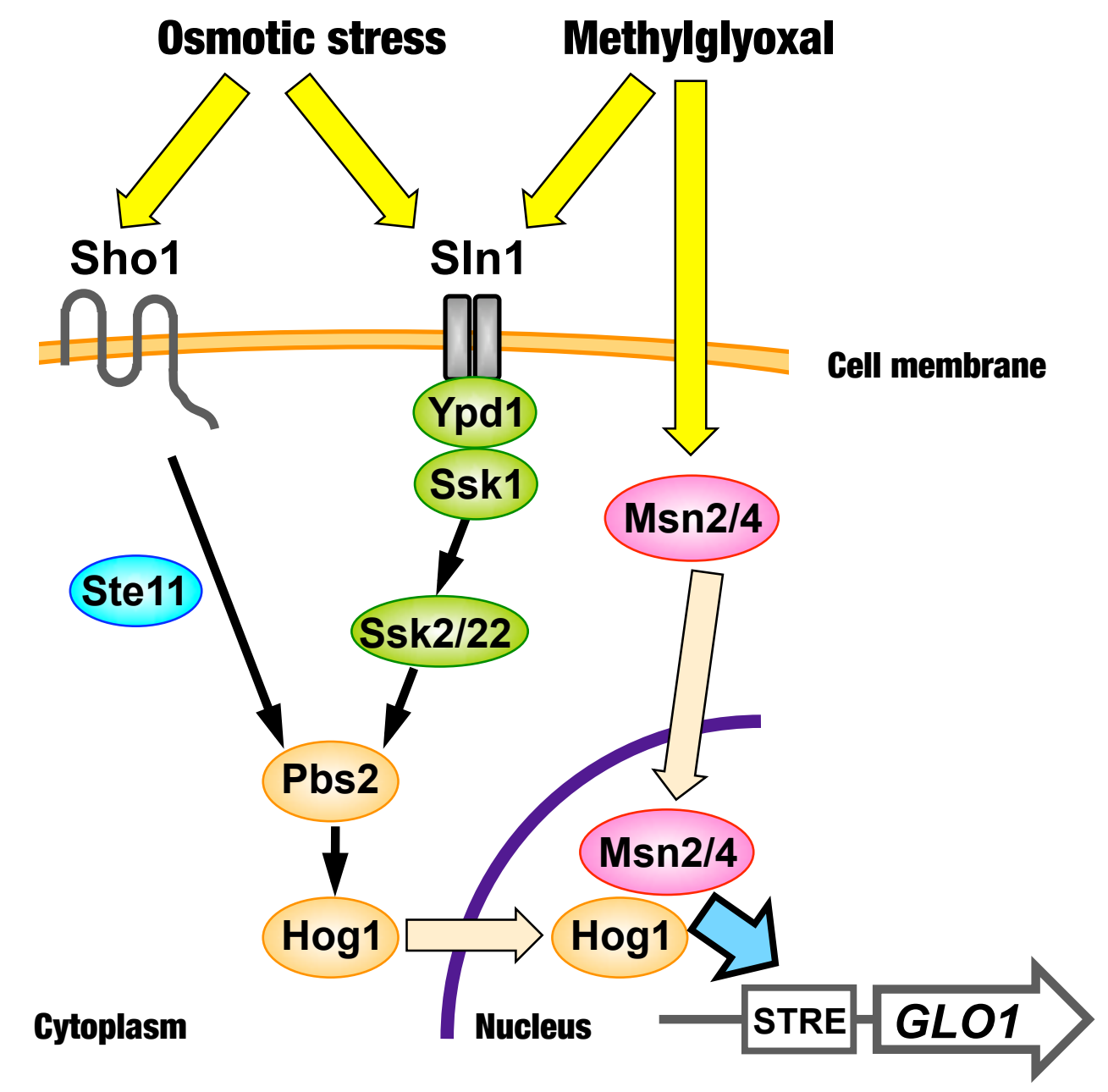


Fig. 2

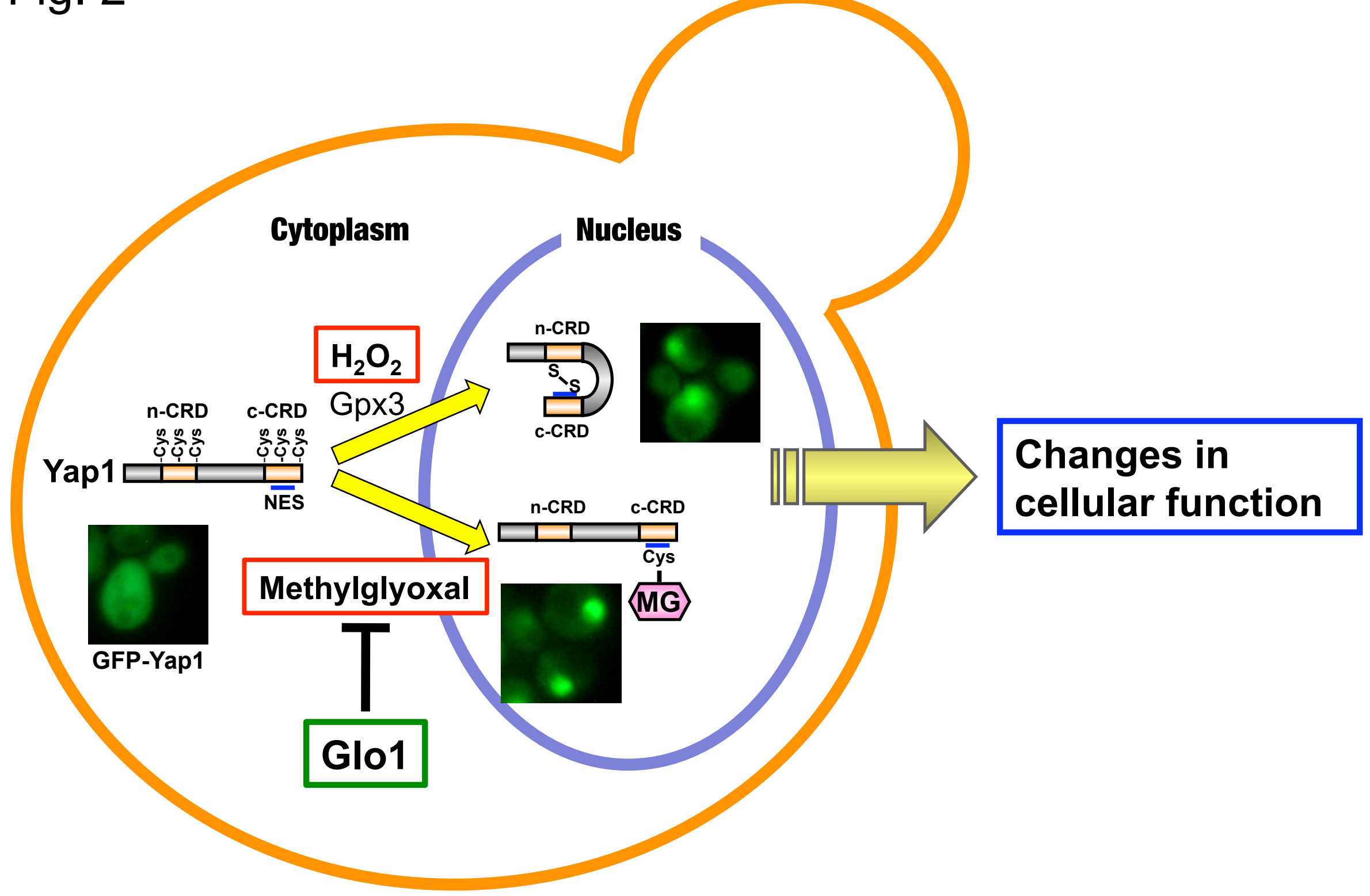

